\title{
Production and Quality Assurance of Main Busbar Interconnection Splices During the LHC 2008-2009 Shutdown
}

\author{
F. Bertinelli, L. Bottura, J.-M. Dalin, P. Fessia, R. H. Flora, S. Heck, H. Pfeffer, H. Prin, C. Scheuerlein, \\ P. Thonet, J.-P. Tock, and L. Williams
}

\begin{abstract}
The main busbar interconnection splices of the Large Hadron Collider are assembled by inductive soldering of the Rutherford type cables and the copper profiles of the stabilizer. Following the September $\mathbf{2 0 0 8}$ incident, the assembly process and the quality assurance have been improved, with new measurement and diagnostics methods introduced. In the 2008-2009 shutdown the resistance both in the superconducting and in the normal conducting states have been the focus for improvements. The introduction of gamma radiography has allowed the visualization of voids between cable and stabilizer. It is now known that during the standard soldering heating cycle solder is lost from the busbar extremities adjacent to the splice profiles, leaving parts of the cable in poor contact with the stabilizer. A room temperature resistance measurement has been introduced as a simple, non-destructive test to measure the electrical continuity of the splice in its normal conducting state. An ultrasonic test has been performed systematically in order to verify if the vertical gaps between the splice profiles are filled with $\mathrm{Sn96Ag4}$ solder. Visual inspections of the different splice components before and after interconnection have been reinforced. The additional information gained has allowed targeted improvements in the splice production process. Ad-hoc machining of splice components avoids macroscopic gaps, additional soldering foil and copper shims are used in critical areas in order to improve the cable to stabilizer contact.
\end{abstract}

Index Terms-Busbars, interconnections, resistance measurement, superconducting cables.

\section{INTRODUCTION}

$\mathbf{T}$ HE 10170 main busbar interconnection splices of the Large Hadron Collider (LHC) [1] are assembled by soft soldering of the Rutherford type cables, of the splice copper profiles (so-called U-piece and wedge) and of the adjacent busbar stabilizer, using lead free $\mathrm{Sn} 96 \mathrm{Ag} 4$ solder and non-activated rosin liquid flux Kester 135.

A LHC main interconnect splice is shown in Fig. 1. After solder connection of the two opposing cables and the splice $\mathrm{Cu}$ stabilizer profiles, the cross section through a splice resembles the adjacent busbar cross section [2], [3].

Manuscript received August 01, 2010; accepted October 01, 2010. Date of publication November 09, 2010; date of current version May 27, 2011.

F. Bertinelli, L. Bottura, J.-M. Dalin, P. Fessia, S. Heck, H. Prin, C. Scheuerlein, P. Thonet, J.-P. Tock, and L. Williams are with CERN, 1211 Geneva, Switzerland (e-mail: Christian.Scheuerlein@ cern.ch).

R. H. Flora and H. Pfeffer are with Fermilab, Batavia, IL 60510 USA.

Color versions of one or more of the figures in this paper are available online at http://ieeexplore.ieee.org.

Digital Object Identifier 10.1109/TASC.2010.2085072

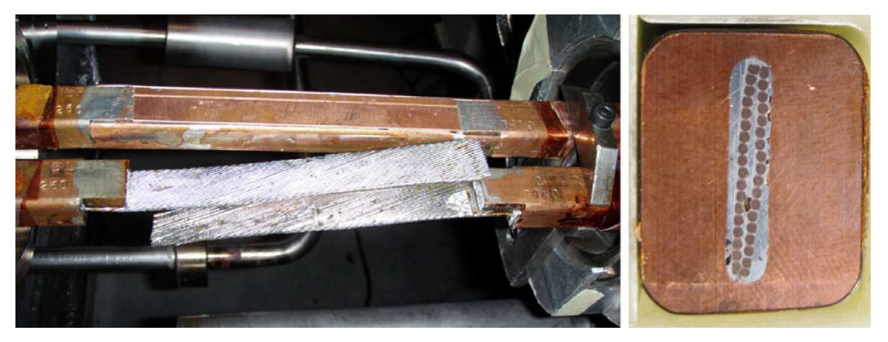

Fig. 1. (a) Main busbar interconnection splices in the LHC tunnel. The splice in the background is finished and in the foreground the two Rutherford type cables extending from the opposing busbar stabilizers are prepared for interconnection. (b) Cross section through a $\mathrm{LHC}$ dipole busbar. The $\mathrm{Nb}-\mathrm{Ti} / \mathrm{Cu}$ cable is electrically connected with the $\mathrm{Cu}$ stabilizer through the $\mathrm{Sn} 96 \mathrm{Ag} 4$ solder alloy.

For normal operation, the splice electrical resistance in the superconducting state of approximately $0.3 \mathrm{n} \Omega$ is the key parameter. However, the resistance of the $\mathrm{Cu}$ stabilizer joint becomes of utmost importance following a magnet quench while the energy stored in the magnets is extracted in dump resistors.

Following the September 2008 incident, the assembly process and the quality assurance have been improved, with new measurement and diagnostics methods introduced. The quality control (QC) tests performed on the finished splices include room temperature (RT) electrical resistance measurements, gamma radiography, and ultrasonic (US) testing. In this article the improvements in the splice assembly process and the QC methods are described, and the main QC test results are summarized. The QC results described in this article complement so-called noninvasive splice tests that can be performed without opening the LHC interconnections, and which are described elsewhere [4].

\section{Improvements of the Splice Production Process}

During the assembly of the main interconnection splices in the 2008-2009 shutdown, particular emphasis has been on the avoidance of macroscopic gaps between splice $\mathrm{Cu}$ profiles and the busbar stabilizer. Production of splices without macroscopic gaps has been achieved by careful alignment of the splice components and, in few cases, by ad-hoc machining of splice components.

Additional soldering foil and copper shims have been used in critical areas of the splice in order to improve the cable to stabilizer contact.

The soldering temperature cycle has been measured and recorded by two independent systems in order to exclude the possibility of an underheating of the splice extremities. 

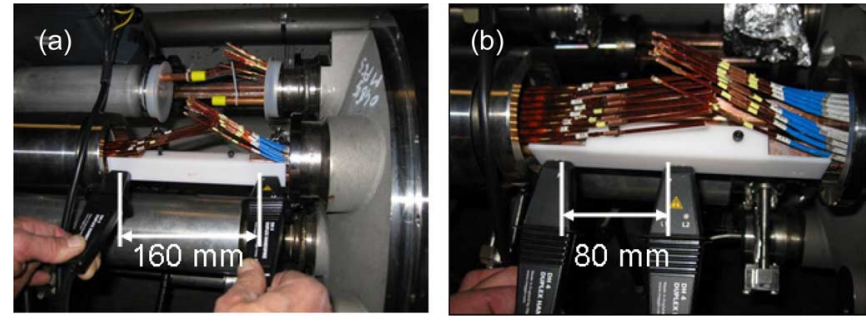

Fig. 2. (a) R-16 and (b) R-8 measurement with a digital low resistance ohmmeter at a quadrupole splice of a LHC interconnection.

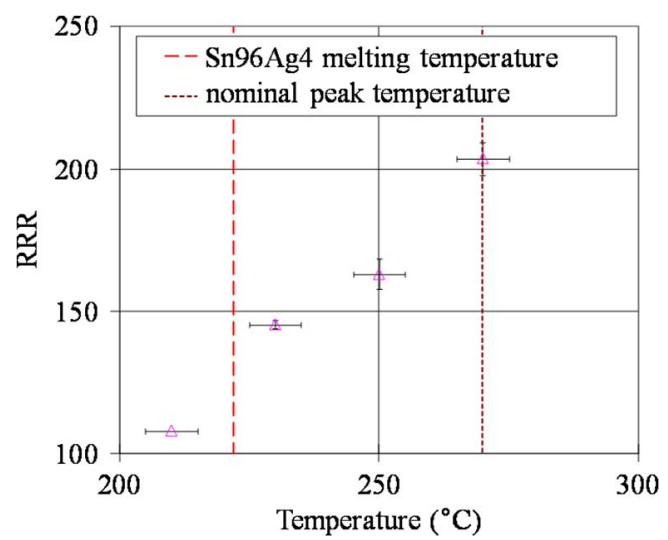

Fig. 3. RRR evolution vs. soldering peak temperature of strand extracted from a LHC busbar cable. The dotted lines indicate the Sn96Ag4 melting temperature and the nominal peak temperature of the LHC main busbar soldering cycle.

\section{RoOM TEMPERATURE ELECTRICAL RESISTANCE RESUlTS}

Room temperature splice resistance measurements have been introduced during the shutdown 2008-2009 in order to detect splices with an excessive resistance in the normal conducting state. Four-point resistance measurements are performed with a Digital Low Resistance Ohmmeter (Avo DUCTER DLRO 10) either with a voltage tap distance of $8 \mathrm{~cm}$ or $16 \mathrm{~cm}$ (referred to as R-8 and R-16, respectively). The R-16 result includes the resistance of both busbar to splice contacts, while the R- 8 result is influenced by one contact only. Since at the LHC interconnects the busbar length that can be accessed is limited (see Fig. 2), the current of $10 \mathrm{~A}$ is injected in a distance of only $5 \mathrm{~mm}$ from the voltage taps.

The inhomogeneous current distribution due to the point like current injection close to the voltage taps causes a systematic error in the R-8 and R-16 results of approximately $+10 \%$ [5]. The estimated random error in the R-8 and R-16 measurements is smaller than $\pm 1 \mu \Omega$. Typical R-16 values for good dipole and quadrupole splices are $10.3 \mu \Omega$ and $17.3 \mu \Omega$, respectively.

Assuming that the overall splice resistance in the non-superconducting state is mainly determined by the bulk resistance of the splice $\mathrm{Cu}$ components and a constriction resistance, the splice resistance at cryogenic temperature can be estimated from R-8/R-16 values when the residual resistivity ratio (RRR) of the different splice $\mathrm{Cu}$ parts (cable stabilizer, busbar stabilizer, wedge and U-piece) is known. In Fig. 3 the RRR evolution of a LHC busbar cable strand in the temperature interval of interest for splice soldering is presented. When the cable is heated with

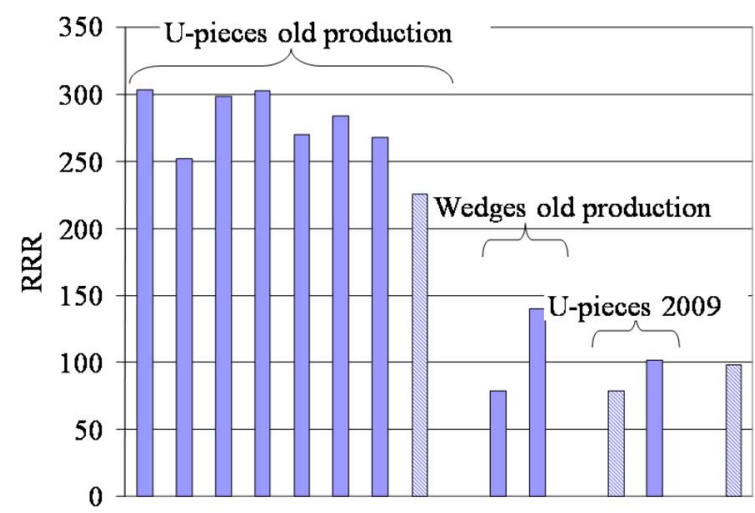

Fig. 4. RRR of different $\mathrm{Cu}$ profiles used for the assembly of the LHC main busbar interconnection splices.

a ramp rate $>100{ }^{\circ} \mathrm{C} / \mathrm{min}$ to the $\mathrm{Sn} 96 \mathrm{Ag} 4$ melting temperature $\left(221^{\circ} \mathrm{C}\right)$ and to the nominal peak temperature $\left(270{ }^{\circ} \mathrm{C}\right)$, the RRR increases to about 130 and 200, respectively. The RRR values found for some components (U-pieces and wedges) that have been used for the assembly of LHC splices are shown in Fig. 4 [6].

The RT and cryogenic resistance measurements for $\mathrm{Sn} 96 \mathrm{Ag} 4$ soldered splices obtained so far in the laboratory gave a R-RT/R-77 K resistance ratio of between 7.3 and 9 [5], and a R-RT/R-10 K resistance ratio between 100 and 300 [7], corresponding with the expected RRR range of the different splice components.

In Fig. 5 the distribution of additional resistances is compared for selected "old" quadrupole (M1+M2) and dipole (M3) splices that have been produced before 2009, and for all "new" splices produced during the 2008-2009 shutdown. The additional resistance is defined as the measured R- 8 value minus an average R-8 value of $9.3 \mu \Omega$ and $5.6 \mu \Omega$ that has been determined for good quadrupole and dipole splices, respectively (for comparison, the resistance values calculated for a $8 \mathrm{~cm}$-long continuous quadrupole and dipole busbar with an assumed $\mathrm{Cu}$ resistivity of $\rho_{\mathrm{Cu}}=1.724 \times 10^{-8} \Omega \mathrm{m}$ are $8.6 \mu \Omega$ and $4.9 \mu \Omega$, respectively).

The maximum additional R- 8 resistance that has been measured for the 2009 production of M1, M2 and M3 splices is 2.8 $\mu \Omega, 6.2 \mu \Omega$ and $1.6 \mu \Omega$, respectively. Much higher resistance values have been found for some "old" splices produced before 2009. A pre-selection of the LHC interconnections that were opened in order to perform R-8/R-16 measurements of "old" splices has been done based on results of the so-called non-invasive resistance measurements [4]. Therefore, the resistance results presented in this article for the splices produced before 2009 are not representative for the entire LHC splice population.

A comparison between R-8 values measured at the lyra side and connection side (see Fig. 6) shows that for "old" M1 and M3 splices most excess values $>5 \mu \Omega$, and all excess values $>30 \mu \Omega$ are found on the lyra side. This might be caused by the asymmetry of the induction heating and splice compression tooling. A systematic difference in the resistance at the lyra and connection side of M2 splices was not observed from the analysis of the few test results for M2 splices with high excess resistance. 

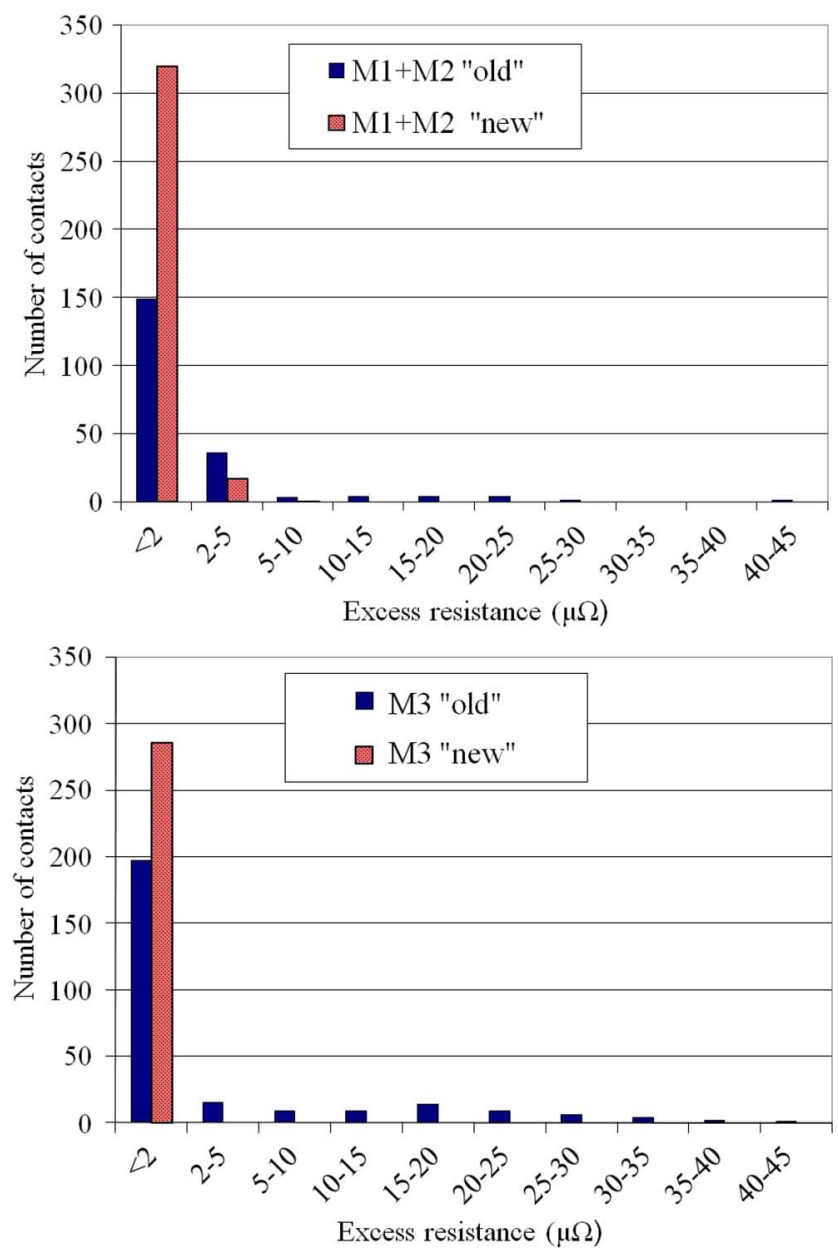

Fig. 5. Distribution of additional R-8 resistances of quadrupole (M1+M2) and dipole (M3) splices produced before 2009 ("old") and during 2009 ("new").

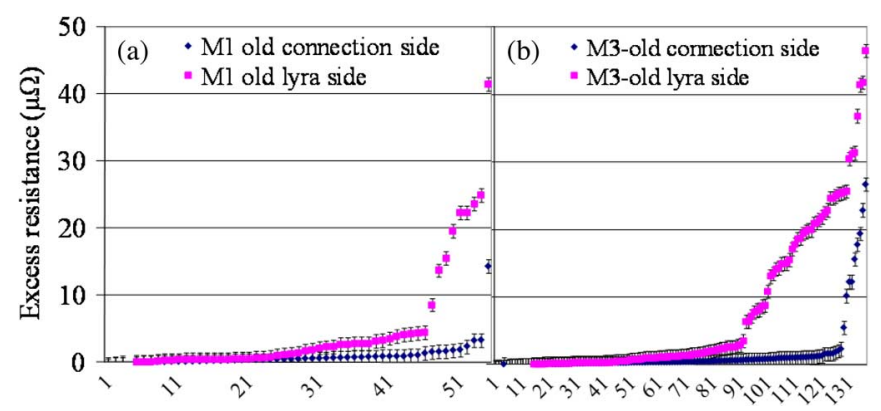

Fig. 6. Distribution of additional R- 8 values for (a) "old" M1 and (b) M3 splices at the lyra and connection side ordered by the additional resistance. The error bars indicate the estimated random error in the resistance measurements $(<$ $\pm 1 \mu \Omega)$.

The maximum additional R-8 resistance measured for dipole splices is $47 \mu \Omega$. All defects for which an additional resistance $>30 \mu \Omega$ has been measured are on the lyra side. From a statistical analysis of the R-16 results obtained for "randomly" tested splices produced before 2009 , it is estimated that about $15 \%$ of the LHC splices have an additional RT resistance $>10 \mu \Omega$ and need consolidation [8], [9].
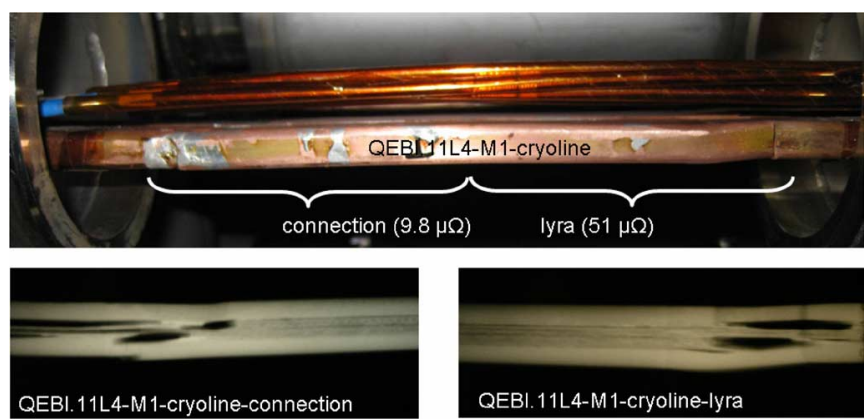

Fig. 7. Image of quadrupole splice with high excess resistance $(+42 \mu \Omega)$ at the lyra side. Gamma ray images reveal lack of Sn96Ag4 solder in the adjacent busbars.

\section{GAMMA RADIOGRAPHY RESUlTS}

Radiographic tests of the $20 \mathrm{~mm}$-thick busbar splices using a Selenium 75 source have been introduced in February 2009. From the absorption contrast in the gamma ray images it has been possible to identify two splice internal defect types.

\section{A. Lack of Solder in the Busbar Adjacent to the Splice}

In the LHC busbars, current sharing between the superconducting cable and the surrounding busbar stabilizer is assured through the Sn96Ag4 solder alloy between cable and stabilizer [see Fig. 1(b)]. Gamma ray images of finished splices show that in some cases solder alloy is lost during the soldering process at the busbar extremities. The nominal peak temperature (measured in the splice center) is $270{ }^{\circ} \mathrm{C}$. Through conduction, the busbars adjacent to splice are heated up to a temperature above the $\mathrm{Sn} 96 \mathrm{Ag} 4$ melting temperature of $221{ }^{\circ} \mathrm{C}$, which causes the loss of Sn96Ag4 in the busbars.

If together with the loss of solder alloy there is a gap between the splice $\mathrm{Cu}$ profiles and the busbar stabilizer, this causes an additional resistance that is nearly proportional to the resistance of the busbar cable over its isolated length (about $13 \mu \Omega$ per $\mathrm{cm}$ isolated cable).

This defect has been the reason for an additional resistance of about $40 \mu \Omega$ of the splice shown in Fig. 7. On both sides of the quadrupole splice, lack of $\mathrm{Sn} 96 \mathrm{Ag} 4$ is visible in the gamma ray images. In addition, at the so-called "lyra side" of the connection a complete gap between the $\mathrm{Cu}$ profiles is present (this gap is not visible in the gamma ray images). After un-soldering and re-soldering the splice the overall splice resistance has been reduced from $\mathrm{R}-16=60.4 \mu \Omega$ to $\mathrm{R}-16=19.6 \mu \Omega$, despite the fact that the void space between cable and stabilizer had not been re-filled with solder alloy.

\section{B. Unmolten SnAg Solder Alloy at the Splice Extremities}

Gamma ray images revealed that in some splices at the extremities unmolten $\mathrm{Sn} 96 \mathrm{Ag} 4$ foil was present, indicating that during the soldering process the $\mathrm{Sn} 96 \mathrm{Ag} 4$ melting temperature was only reached in the splice center, but not at the extremities. In the gamma ray images shown in Fig. 8, the bright areas are those made of the most absorbing material in the splice (Sn96Ag4) and the dark areas represent voids.

In addition to these two defect types, the approximately 15 $\mathrm{mm}$-long voids that can be seen in the gamma ray images shown 



Fig. 8. Gamma ray images of the dipole splices (a) QBBI.A25L4-M3-croline-lyra side and (b) QBBI-A30L2-M3-corridor-lyra-side. The R-8 excess resistance of both splices was $31 \mu \Omega$.

in Fig. 8 are present at both extremities of most main busbar splices. At this location between the busbar stabilizer nose and the splice U-piece the space for two cables is filled by one cable only, leaving systematically voids between busbar stabilizer and cable.

The RT resistance measurements of the corresponding splices indicate that there is hardly any current sharing between the cable surrounded by unmolten Sn96Ag4 foil and the busbar stabilizer. Therefore, this defect type can cause high additional splice resistances. Most of the defects with R- 8 excess resistances $>30 \mu \Omega$ that have been detected are of the second type (unmolten solder in the splice extremities).

\section{UltRAsOnic TeSt Results}

An Ultrasonic (US) test had already been introduced at the end of the series LHC interconnection work before 2009. A detailed description of the standard US test can be found in [10]. All splices produced during the 2008-2009 shutdown have been US tested. During the standard test, the US transmission through the $\mathrm{Cu}$ U-piece and the $\mathrm{Cu}$ wedge of the splice is measured in four equidistant positions across the splice. Only in some cases, the transmission through the U-piece and the stabilizer nose has been measured too (see Fig. 9).

If the transmitted US amplitude exceeds a threshold value in a certain time window, the test result is classified as "OK" otherwise as "NOT OK". In the standard test configuration a good US transmission indicates that the gaps between U-piece and wedge have been filled by solder alloy. Thus, if the US transmission is "OK", it can be concluded that solder foil had been added when the splice was assembled and that the Sn96Ag4 melting temperature had been reached during the subsequent heating cycle.

In addition to the US reflections inside the splice, in the test configuration described above the US amplitude is also influenced by variations of the contact pressure between transducer

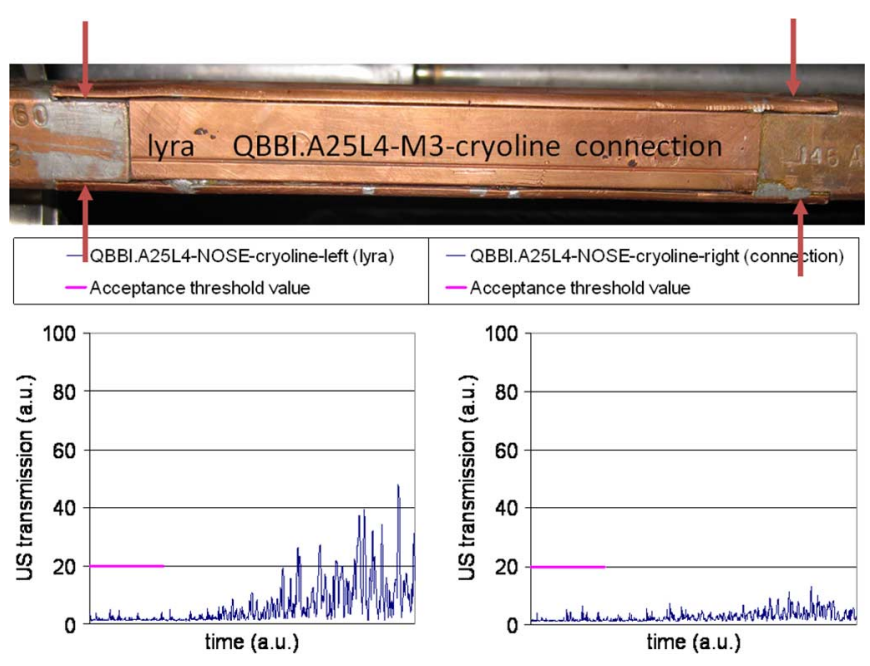

Fig. 9. US test approximate positions for measuring the transmission through U-piece and stabilizer nose (splice "old" production).
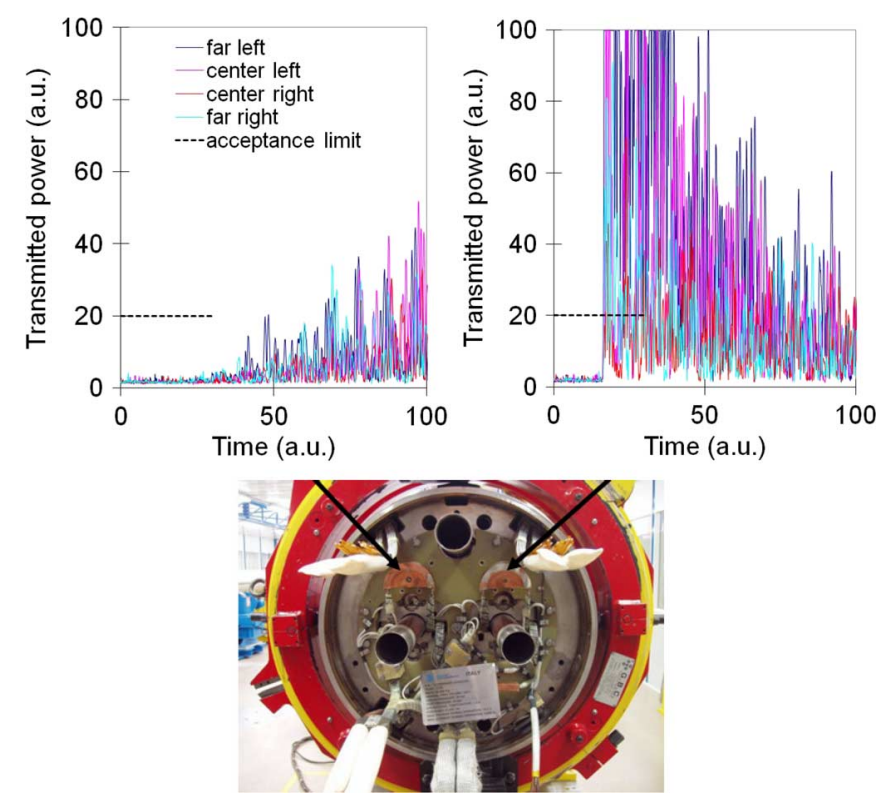

Fig. 10. US transmission test result for a high-resistance (left) and low-resistance (right) interpole splice in cryodipole 2334.

and splice, and by variations of the splice surface state. Therefore, a quantitative comparison of US test results is difficult. Nevertheless, a splice without filler material can be clearly distinguished from a good splice.

The usefulness of the US transmission test for detecting splices in which filler material was omitted and/or the $\mathrm{Sn} 96 \mathrm{Sg} 4$ melting temperature was not reached has been confirmed by testing two high resistance splices in two LHC cryodipoles. These splices had been produced during cold mass assembly, and were identified by calorimetric tests in the LHC tunnel. Later examination revealed that the reason for the high resistance in these splices was a lack of filler material. As can be seen in Fig. 10, in all 4 measurement positions of the left (high resistance) splice of cryodipole 2334 the transmitted amplitude remained clearly below the threshold value, while in the right 
splice (produced with filler material) in all 4 test positions the US power exceeded the threshold value.

Out of the 208 splices produced before 2009 that were US tested during the 2008-2009 shutdown, 10 splices were repaired because the US test result in one out of four standard test positions was "NOT OK". The additional US test (US transmission between U-piece and stabilizer nose) failed in many cases. This may be explained by the fact that the mechanical distortion of the splice profiles assembled before 2009 is particularly strong at the splice extremities. In some cases the poor US transmission may also indicate that at the splice ends the Sn96Ag4 melting temperature was not exceeded during the soldering cycle.

For all splices with an additional resistance $>10 \mu \Omega$ for which the additional US test at the splice noses has been performed, it was found that at the high resistance side of the splice the US test between U-piece and stabilizer nose failed. As an example, the additional R-8 resistances for the splice shown in Fig. 9 are $+8.5 \mu \Omega$ and $+27 \mu \Omega$ at the connection and lyra side, respectively, and the additional US test failed at both sides. It appears that a good US transmission through the U-piece and the stabilizer noses is an indication for the electrical continuity of the $\mathrm{Cu}$ stabilizer through the splice.

Due to the improvements in the splice assembly process that are described above, for new splices produced during 2009 the US test results are more homogenous. The standard US test of only one out of a total of 332 splices analyzed failed. The reason for the single failed test was that a wrong $\mathrm{Cu}$ wedge had been mounted (M1 type instead of M3 type). After splice repair the US test was passed.

The transmission through U-piece and busbar stabilizer noses was measured for 54 new splices, and in all cases the US test result was clearly passed, showing the beneficial effect of the more precise U-piece machining and alignment procedures, and possibly the more rigorous temperature control introduced during 2009.

\section{DISCUSSION AND CONCLUSION}

Room temperature resistance measurements have been found to be an excellent tool for the quality control of the LHC main busbar splices. The ratios of splice resistances at RT and cryogenic temperatures (above the critical temperature of the $\mathrm{Nb}-\mathrm{Ti}$ alloy) obtained in the laboratory for several Sn96Ag4 soldered splices with varying defect resistance indicate that the low temperature resistance can be roughly estimated from R-8/R-16 values when the RRR of the splice components is known.

The benefits of the improvements in the splice production process that were introduced during the 2008-2009 shutdown are clearly revealed by the comparison of RT resistance results achieved for splices produced before and during 2009 (see Fig. 5).

The standard US test allows to detect splices in which no solder foil had been added and/or for which the solder melting temperature had not been reached during production. A comparison between the US transmission through splice U-pieces and busbar stabilizer noses with $\mathrm{R}-8$ results indicates that the
US test can be useful beyond its initial purpose, in order to conclude on the electrical continuity of the $\mathrm{Cu}$ stabilizer through the splice. However, a direct 4 point RT resistance measurement is preferable for this purpose, because it can provide a quantitative result, which for splices with large defects is nearly proportional to the defect size.

The introduction of gamma ray imaging has allowed to identify two volume defect types in high resistance splices, notably lack of Sn96Ag4 alloy in the busbar stabilizer extremities in combination with a gap between the $\mathrm{Cu}$ profiles, and un-molten Sn96Ag4 alloy at the splice extremities. Despite the very valuable information gained, gamma radiography cannot be considered as a tool for the routine QC of LHC splices, since even high quality gamma ray images do not allow to conclude on the absence of planar flaws that are smaller than the spatial resolution of the technique (in the present case in the order of $0.1 \mathrm{~mm}$ ).

The newly introduced QC tests that can be performed at room temperature allow to conclude on the electrical continuity of the busbar stabilizer across the splice. However, from the room temperature tests the presence of a damaged superconducting cable and/or a bad cable-to-cable contact inside the splice cannot be excluded. In order to avoid high resistance splices to be found later on during resistance measurements at cold, careful visual inspections of the splice components prior to splice assembly are needed. The most important non-conformities concerning the superconducting cables found during the 2008-2009 shutdown had been identified by the teams producing the electrical interconnections, demonstrating that quality assurance relies strongly on the skill and commitment of the personnel performing the electrical interconnection work.

\section{REFERENCES}

[1] The Large Hadron Collider; A Marvel of Technology, L. Evans, Ed.. Lausanne/Boca Raton, FL: EPFL/CRC Press, 2009.

[2] L. Belova, M. Genet, J.-L. Perinet-Marquet, P. Ivanov, and C. Urpin, "Design and manufacture of the superconducting bus-bars for the LHC main magnets," IEEE Trans. Appl. Supercond., vol. 12, no. 1, pp. 1305-1309, 2002.

[3] A. Jacquemod, A. Poncet, F. Schauf, B. Skoczen, and J. P. Tock, Inductive soldering of the junctions of the main superconducting bus bars of the LHC CERN-LHC-Project-Report-698, 2004.

[4] M. Koratzinos et al., "High-current bus splice resistances and implications for the operating energy of the LHC," in IPAC 2010 proceedings.

[5] S. Heck, L. Dufay-Chanat, and C. Scheuerlein, Resistance of LHC main busbar splices at room temperature and at 77.4 K CERN TE-MSC Technical Note, EDMS Nr. 1053983, 2009.

[6] S. Heck, C. Scheuerlein, P. Fessia, and R. Principe, The RRR of the $\mathrm{Cu}$ components of the LHC main busbar splices CERN TE-MSC Technical Note, EDMS Nr. 1057918, 2010.

[7] G. P. Willering, L. Bottura, P. Fessia, G. Peiro, S. Le Naour, R. Principe, T. Renaglia, D. Richter, C. Scheuerlein, and A. Verweij, "Thermal runaways in LHC interconnections experiments," in these proceedings.

[8] F. Bertinelli et al., "Towards a consolidation of LHC superconducting splices for $7 \mathrm{TeV}$ operation," in IPAC 2010 proceedings.

[9] A. P. Verweij et al., "Consolidation of the $13 \mathrm{kA}$ interconnects in the LHC for operation at $7 \mathrm{TeV}$," in these proceedings.

[10] F. Caspers, T. Kroyer, J. Kulka, J.-P. Tock, and L. R. Williams, Ultrasound diagnostics of the superconducting cable connections between the main ring magnets of LHC LHC-Project-Report-1032, 2007. 